\title{
"¡ME DOY CUENTA DE QUE ELLA TIENE MENTE...!" Una reflexión sobre el papel de la maternidad en un contexto de duelo ${ }^{1}$
}

\author{
María Isabel Pérez Rigau² \\ Castelldefels, Barcelona
}

En general, existe una extensa bibliografía sobre los efectos del estado de ánimo de la madre sobre el desarrollo del bebé pero son menores los estudios que se centran en los efectos de la parentalidad en general y la maternidad en particular, sobre los progenitores. A partir de la exposición de un caso clínico, se plantea una reflexión sobre el papel de la maternidad en un proceso de duelo. La perspectiva intersubjetiva amplifica la comprensión sobre las interacciones relacionales; el reconocimiento del otro y la construcción del sentido de nosotros, de "nostredad", como fundamento del sentimiento de identidad.

Palabras clave: Intersubjetividad, duelo, maternidad, reconocimiento, "nostredad", identidad.

In general, there is extensive literature on the effects of the mother's mood on the baby's development but the ones that focus on the effects of parenthood in general and motherhood in particular, on the parents are few. From the exposition of a clinical case, a reflection on the role of motherhood in a grieving process arises. The intersubjective perspective amplifies the understanding of relational interactions; the acknowledgement of the other and the creation of a feeling of "Weness" as the foundation of the sense of identity.

Key Words: Intersubjectivity, mourning, motherhood, acknowledgement, "We-ness", identity.

English Title: "I'm aware of she has mind..." A reflection on the role of motherhood in a context of grieving

\section{Cita bibliográfica / Reference citation:}

Pérez Rigau, I. (2016). "¡Me doy cuenta de que ella tiene menta...!" Una reflexión sobre el papel de la maternidad en un contexto de duelo. Clínica e Investigación Relacional, 10 (1): 106-112. [ISSN 1988-2939] [Recuperado de www.ceir.info ] DOI: 10.21110/19882939.2016.100108

\footnotetext{
${ }^{1}$ Comunicación presentada en la 6a Reunión Anual de IARPP-España, Valencia, Octubre 2015

2 Psicóloga Especialista en Psicología Clínica. Dirección de contacto: C/ Alacant, 4. Baixos $2^{\circ}$. 0886oCastelldefels. Barcelona.
} 
"Me doy cuenta de que ella tiene mente..."; "Ahora miramos juntas los colores...; "Estuvimos mirando juntas las flores....". Así me relataba Gloria los cambios que observaba en la interacción con su con su hija Calma, de dos años. Nacida unos meses después de la muerte de su padre, por suicidio. En este "mirar juntas" se condensaban los cambios que empezaban a observarse en la relación.

Los estudios de Stern (1977 - 2008) sobre las relaciones madre - bebé nos han facilitado una mayor reflexión sobre las interacciones y su repercusión en el desarrollo del niño. Anteriormente los trabajos de Esther Bick (1968) sobre observación de bebés nos brindaron la oportunidad de iniciar una reflexión, no sólo sobre la relación madre - bebé sino también el papel de la observación en la intervención psicoterapéutica. Desde una perspectiva clínica, si bien el post parto ha sido considerado como una etapa de riesgo e incremento de la clínica en pacientes, por ejemplo con alteraciones del estado de ánimo, el embarazo aparece en algunos estudios como un factor protector ante determinadas patologías (Grof et alt. 2000). En general, existe una extensa bibliografía sobre los efectos del estado de ánimo y salud de la madre sobre el desarrollo posterior del bebé (Fonagy 1991) pero son menores los estudios que se centran en los efectos de la paternidad en general y, la maternidad en particular, sobre el desarrollo emocional y psicológico de los propios progenitores. En esta dirección, Stern (2008) profundiza en el mundo interno de la nueva madre y la influencia de la maternidad en su estructura mental. En esta nueva realidad convergen emociones de pérdida - dejar de ser sólo hija - y experiencias positivas que permitirán, si el desarrollo es normal, el desarrollo de una nueva identidad.

Este es el objetivo de esta comunicación donde uno de los aspectos más importantes de la intervención psicológica se ha centrado en la maternidad como motor de cambio y de elaboración; como camino de reparación y crecimiento, en un contexto difícil de duelo. Se pretende también una reflexión, desde una perspectiva intersubjetiva, sobre la relación entre los aspectos clínicos y de construcción de identidad.

Gloria fue atendida, en una primera fase, en un centro público del sistema nacional de salud. Siendo remitida por su médico de cabecera por Alopecia Areata y síntomas depresivos con ansiedad que fueron valorados en un primer momento como reactivos a situación laboral. Fue atendida durante poco más de un año con una periodicidad quincenal. De su historia personal destacamos el fallecimiento de un hermano cuando ella tenía dos años, separación del núcleo familiar en este período y diagnóstico de leucemia de su hermano menor al volver al núcleo familiar. Durante la terapia se valoró episodio puntual con alteraciones a nivel de 
pensamiento, omnipotencia y pensamiento mágico. Este episodio se produce en contexto de crisis de pareja de la que después plantea separarse por problema de enolismo de él. A su vez, diagnóstico de enfermedad venérea del padre de la paciente que genera importante crisis entre sus progenitores. Se valoraron también los rasgos perfeccionistas y de exigencia en la personalidad de la paciente. "Ser perfecta para no sentir vergüenza", el conocimiento relacional implícito que se trabajó en esta primera etapa de tratamiento.

Tras mejora sintomática y trascurrido un año, se dejó a iniciativa de la paciente establecer nuevo contacto y ésta lo reanudó más de un año después, tras fallecimiento por suicidio de la pareja y siendo gestante de 6 meses. Explica entonces que él estuvo recibiendo tratamiento en un centro hospitalario por presentar un brote psicótico.

En una primera fase de la terapia el Duelo parece ocuparlo todo. No permite inicialmente soñar el bebé, no parece construirse una matriz inicial que permita la construcción de una identidad como madre o si se construyó, quedó interrumpida. Siguiendo a Stern, la madre parece mantener un "patrón de apego superficial". Mucha distancia en su experiencia de embarazo. No puede reportar sensaciones, ensoñaciones, vivencias que traduzcan un vínculo autónomo. Poco a poco "patrón de apego implicado", búsqueda de su propia madre como referencia. Todo lo bueno y positivo puesto en ella y lo malo en "la madre de él". En este primer período, pienso a menudo que no existe lugar para el bebé. Tras el nacimiento de Calma, la identidad materna se instala con mucha lentitud. Como terapeuta pienso en el tratamiento como facilitador de la interacción pero me resulta difícil acompañarla en el dolor y mantener un equilibrio entre el trabajo de duelo y hacer presente al bebé. Me siento muy pendiente de validar la experiencia como madre, marcar muy de cerca sus estados emocionales. A menudo, tengo la sensación de que el bebé deberá luchar para captar la atención de la madre. Calma "despierta ternura" con su nacimiento, dice ella. Da la impresión de que el embarazo se prolonga durante todo el primer año de vida de Calma. Como si fuera difícil diferenciar el sí mismo, donde "no hay sitio para dos" (Wallin 2012), sin posibilidad de identidad diferenciada. Pero Calma revitaliza a su madre y la madre responde, mediante cursos y actividades- curso de nutrición, natación, estimulación...- Que le permiten estar más cerca del bebé pero con límites tolerables para ella. La madre puede expresar que "Miro y observo a Calma" pero no poder acercarse demasiado por "temor a encariñarme y perderla". Tiene miedo de que la niña herede la enfermedad del padre, probable esquizofrenia.

Al final del $2^{\circ}$ año de la niña, se inicia un período con mucho conflicto de Gloria con su propia madre, muy ambivalente, donde confluyen: reconocimiento - preguntándose qué debió sentir su madre cuando falleció su hermano - y, a la vez, queja- cuando la percibe muy 
exigente, incluso "cruel"... A su vez, la abuela parece percibir a su hija demasiado exigente con la niña. La trata demasiado de mayor, "demasiado independiente", dice. Reporta una serie de situaciones que me hacen pensar que sobre Calma recae el papel de tirar de la madre- "En un $80 \%$ lo programo todo por ella, yo no haría casi nada... Siempre está contenta..." "Si ella tira...yo tiro..."

Duelo y maternidad parece que pueden integrarse cada vez más y poco a poco en el espacio de entrevista. Gloria puede reportar escenas muy dolorosas vividas en relación al suicidio de su pareja. Pero ahora quiere que su hija aprenda el idioma del padre -inglés- para poder comunicarse con su abuela paterna, hasta hace poco percibida como figura negativa y con rencor... Su propia madre, la abuela materna, puede ser ahora cuestionada. Tolerando mejor la ambivalencia que puede ser trabajada y que permite poder mantener una matriz madre bebé - abuela que facilite el desarrollo de Calma. Así es como la abuela señala que Gloria trata a su hija como niña mayor y no lo es... Gloria dice que su madre no se deja ayudar con su padre - diagnóstico de demencia-. Calma no quiere que su abuelo le obligue a dibujar donde él quiere...Calma dice, "no" pero a veces muerde a otros niños en la guardería, los empuja y prefiere estar con niños mayores... Tiene dos años y 8 meses, se viste casi sola... pero necesita el pecho para dormir. Pero los "noes" de Calma ayudan a repensar y modificar a Gloria sus formas de hacer, sus "protocolos a cumplir..."; como lo hizo antes en su lugar de trabajo.

Gloria comenta que se siente mucho mejor: "Me doy cuenta de que ella tiene mente...Antes no lo veía así...Ahora miramos juntas los colores... Estuvimos mirando juntas las flores...". El tratamiento está todavía en proceso pero creo que plantea de nuevo la importancia del reconocimiento del otro como base de desarrollo y evolución; el reconocimiento del otro como alimento del self... Verse a uno mismo como objeto y como sujeto (Benjamin, 1999; Coderch 2012). En este periodo Gloria se muestra, a su vez, con mayor capacidad para poder reportar mayores contenidos en las sesiones. El pasado se hace presente. Puede hablar de sus experiencias anteriores, sus recuerdos, sus relaciones y traer con mayor detalle sus pensamientos y sentimientos entorno al suicidio de su pareja, su sentimiento de culpa...

El dolor no es la patología, dice Stolorow (2012), es la ausencia de una sintonía adecuada con las reacciones emocionales dolorosas lo que las hace duraderas $y$, en consecuencia, son fuente de estados traumáticos y de psicopatología. Gloria presentó patología: Alopecia Areata, Trastorno Adaptativo de tipo depresivo, episodio muy breve con alteración de pensamiento de contenidos mágicos... Pero a pesar de que disociación, negación y tendencia a las respuestas de tipo omnipotente creo que han jugado un papel importante en su funcionamiento, ahora pude empezar a pensar más en sí misma y en los demás de otra 
manera: Puede mentalizar, en el sentido de representarse mejor su propia experiencia (Fonagy 2004, Coderch 2012, Lanza 2013). Ha podido trasformar algunas cosas; pudo "vaciar el trastero con ayuda de un amigo"y pasar de decir en relación a Calma: "Si ella tira, yo tiraré..." a "Ahora tiramos las dos...".

Este trabajo me ha llevado también a repensar en cómo las pérdidas no mentalizadas detienen el desarrollo; En la compañía del otro, como facilitador de mayor integración; en como los cambios positivos en un miembro de la familia generan cambios en la interacción que a su vez facilitan la evolución en otros - la abuela paterna comunica que también ha podido dejar las cenizas de su hijo... "Calma -dice su madre- es una niña que tiene en cuenta al grupo...que se da cuenta de muchas cosas..."- En la importancia de la superación de los sentimientos culposos, sin quedar encallados en la tristeza, ni angustiados por lo no resuelto... En el diálogo entre lo interno y externo como motor evolutivo... En definitiva, la experiencia subjetiva. R Velasco en su artículo Contextos de una Psicoanalista (2011) escribe: El trabajo con los pacientes se beneficia de comprensiones... porque arrancan de la propia experiencia. La línea que separa la diferencia entre paciente y analista se traspasa libremente con el objetivo de avanzar en el desarrollo de identidades con más sentimiento de integración.

A menudo venía a mi mente el recuerdo de mi experiencia en observación de bebés... Concretamente, una imagen en la que la madre sostiene a su bebé en brazos y lo observa a través del espejo. Se miran conjuntamente y, a su vez, observan un cordel que sujeta un objeto que cuelga cerca de ellos. En esa escena pasada recordé los momentos anteriores en los que la conexión entre ellos era más frágil y que a menudo amenazaba una buena evolución. Sin embargo, en ese instante aparecía más sólida y viva... Pensé también en el papel integrativo de la observación en ese momento y ahora aquí, en mi papel con Calma y Gloria, pensando juntas. Recordé los trabajos de Klein sobre la posición depresiva... Reviví/ viví mi identidad de madre y mis propios duelos, que quizá no permitieron tampoco ver al otro, algunas veces... Recordé a Stolorow, la disyuntiva entre la integración por el dolor y la integración del dolor... Pensé en la maternidad y paternidad como marco de integración, diferenciación y creatividad...

Señalaré por último, la complejidad y dificultad del abordaje del trabajo psicoterapéutico en el contexto de Duelo y Maternidad, en difícil equilibrio de intervención. Pero sobretodo, la visión reparadora y creativa de la maternidad, como construcción de una relación desde la intersubjetividad... De Gloria con su hija y también de paciente-terapeuta. "Nosotras" no pudimos tampoco, prever la gravedad del riesgo mental de él... Este nuevo "mirar juntas" parece introducir un sentido de "nosotras", de nostredad (Emde, 2007) que plantea una 
evolución más positiva y esperanzadora y que permite, a su vez, el desarrollo de la propia identidad.

\section{REFERENCIAS}

Ammanti M, Trentini, C. (2009). How new Knowledge about parenting reveals the neurobiological implications of intersubjectivity: A conceptual Synthesis of recent research. PsychoanalyticDialogues, 19:537-555.[Traducción en Aperturas Psicoanalíticas. № 34]

Benjamin, J. (1999). Recognition and destruction: An outline of intersubjectivity. En D. Wallin: El Apego en psicoterapia . Bilbao: Editorial Desclée de Brouwer, 2012.

Bick E. (1968). La experiencia de la piel en las Relaciones de Objeto Tempranas. J of Psychoanalysis,1968, XLI, 2-3

Coderch J. (2010). La Práctica de la Psicoterapia Relacional. El Modelo Interactivo en el Campo del Psicoanálisis. Madrid: Ágora Relacional.

Coderch, J. (2012). Realidad, Interacción y Cambio Psíquico. La Práctica de la Psicoterapia Relacional II. Madrid: Ágora Relacional.

Coderch J. (2012). Identidad, Contexto y Mentalización. Clínica e Investigación Relacional. Vol. 6 (2) Junio 2012; pp. 218-234

Emde,R.N.(2007). Embodiment and our inmersion with others: Commentary on Fonagy and Target. Journal of the American Psychoanalytic Assoc., 55, 485-492

Fonagy $\mathrm{P}$, Steele $\mathrm{H}$ (1999). Maternal representations of attachment during pregnancy predict the organitzation of infant- mather attatchment at one year of age. Child Development,62, 891-905. On line 2008.

Fonagy $\mathrm{P}$, (2004). Teoria del Apego y Psicoanàlisis. Barcelona: Espax

Fonagy P, Gergely G, Jurist E and Target M. (2004). Affect Regulation, Mentalization and the Development of the Self, Londres: Karnac.

Grof et al. (2000). Protective effect of pregnancy in women with lithium - responsive. J Affect Disord ; 61: 31-9

Lanza C, G ( 2013). La mentalización de la afectividad, sus perturbaciones y su abordaje clínico. Temas de Psicoanálisis.

Rodríguez Pereira, C. (2010). Reseña de la obra de Daniel Stern "La constelación maternal". Clínica e Investigación Relacional, 4 (1): 270-312.

Stern, D. (1994). La constelación maternal. Paidos .Barcelona.

Stern, D. et al. (2013). El nacimiento de una madre: Cómo la experiencia de la maternidad cambia la vida para siempre [ Reseña de M. del Castillo] En Aperturas Psicoanalíticas. 44.

Stolorow R, Atwood G., Orange D. (2004). Worlds of Experience, New York: Basic Books

Stolorow R (2011). World, Affectivity and Trauma. New York: Routledge 
Stolorow R (2012). De la Mente al Mundo. Clínica e Investigación Relacional Vol.6(3). Octubre 2012; pp. 381-395

Tronick E, Z (1997). Depressed mothers and infants: Failure to form dyadic states of consciousness.In L. Murray, P.J. Cooper(Eds). Postparum depression and child development (pp.54-88).

Wallin, D. (2012). El Apego en psicoterapia. Bilbao: Editorial Desclée de Brouwer

Original recibido con fecha: 24/10/2015 Revisado: 20/12/2015 Aceptado: 28/02/2016 